\section{Expression of COBLL1 encoding novel ROR1 binding partner is robust predictor of survival in chronic lymphocytic leukemia}

\author{
Hana Plešingerová,, ${ }^{1,2}$ Pavlína Janovská,,$^{3,4}$ Archana Mishra, ${ }^{3}$ Lucie Smyčková, ${ }^{3}$ \\ Lucie Poppová,, ${ }^{1,2}$ Antonín Libra, ${ }^{5}$ Karla Plevová,,,2 Petra Ovesná, ${ }^{6}$ \\ Lenka Radová, ${ }^{2}$ Michael Doubek, ${ }^{1,2}$ Šárka Pavlová, ${ }^{1,2}$ Šárka Pospišilová, 1,2 \\ and Vítězslav Bryja ${ }^{3,4}$
}

\begin{abstract}
${ }^{1}$ Center of Molecular Biology and Gene Therapy, Department of Internal MedicineHematology and Oncology, University Hospital Brno and Medical Faculty, Masaryk University, Brno; ${ }^{2}$ Central European Institute of Technology, Masaryk University, Brno; ${ }^{3}$ Institute of Experimental Biology, Faculty of Science, Masaryk University, Brno;

${ }^{4}$ Department of Cytokinetics, Institute of Biophysics, Academy of Sciences of the Czech Republic, Brno; ${ }^{5}$ Generi Biotech, s.r.o., Hradec Králové and ${ }^{6}$ Institute of Biostatistics and Analyses, Masaryk University, Brno, Czech Republic
\end{abstract}

\section{ABSTRACT}

C hronic lymphocytic leukemia is a disease with up-regulated expression of the transmembrane tyrosine-protein kinase ROR1, a member of the Wnt/planar cell polarity pathway. In this study, we identified COBLL1 as a novel interaction partner of ROR1. COBLL1 shows clear bimodal expression with high levels in chronic lymphocytic leukemia patients with mutated IGHV and approximately $30 \%$ of chronic lymphocytic leukemia patients with unmutated IGHV. In the remaining $70 \%$ of chronic lymphocytic leukemia patients with unmutated IGHV, COBLL1 expression is low. Importantly, chronic lymphocytic leukemia patients with unmutated IGHV and high COBLL1 have an unfavorable disease course with short overall survival and time to second treatment. COBLL1 serves as an independent molecular marker for overall survival in chronic lymphocytic leukemia patients with unmutated IGHV. In addition, chronic lymphocytic leukemia patients with unmutated IGHV and high COBLL1 show impaired motility and chemotaxis towards CCL19 and CXCL12 as well as enhanced B-cell receptor signaling pathway activation demonstrated by increased PLCY2 and SYK phosphorylation after IgM stimulation. COBLL1 expression also changes during $\mathrm{B}$-cell maturation in non-malignant secondary lymphoid tissue with a higher expression in germinal center $B$ cells than naïve and memory B cells. Our data thus suggest COBLL1 involvement not only in chronic lymphocytic leukemia but also in B-cell development. In summary, we show that expression of COBLL1, encoding novel ROR1-binding partner, defines chronic lymphocytic leukemia subgroups with a distinct response to microenvironmental stimuli, and independently predicts survival of chronic lymphocytic leukemia with unmutated IGHV.

\section{Introduction}

Upregulation of transmembrane receptor tyrosine kinase-like orphan receptor 1 (ROR1) in chronic lymphocytic leukemia (CLL) cells was revealed as one of the most stable CLL markers. ${ }^{1,2}$ ROR1 is expressed on the cell surface of patients with mutated (M-CLL) as well as unmutated (U-CLL) IGHV. ROR1 is highly expressed during embryonal development but largely undetectable in the adult organism.,4 Negligible ROR1 expression on healthy B cells ${ }^{4,5}$ makes it a suitable candidate for monitoring CLL remission ${ }^{6}$ and a candidate target for therapy with monoclonal antibodies $^{7}$ or $\mathrm{T}$ cells with ROR1-specific chimeric antigen receptor. ${ }^{8,9}$ Although ROR1 is up-regulated in CLL patients, its activity may vary depending on its posttranslational modification ${ }^{10}$ and on the availability of its dedicated ligands. ${ }^{11}$

ROR1 is a member of the Wnt/PCP (planar cell polarity) signaling pathway, ${ }^{4}$ which regulates various processes during embryonic development, mainly linked to cell polarity, survival and migration. We have previously reported, in accordance

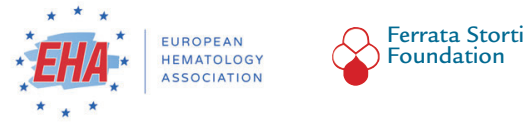

Haematologica 2018

Volume 103(2):313-324

\section{Correspondence:}

bryja@sci.muni.cz

Received: August 18, 2017.

Accepted: November 3, 2017.

Pre-published: November 9, 2017.

doi:10.3324/haematol.2017.178699

Check the online version for the most updated information on this article, online supplements, and information on authorship \& disclosures: www.haematologica.org/content/103/2/313

(C)2018 Ferrata Storti Foundation

Material published in Haematologica is covered by copyright. All rights are reserved to the Ferrata Storti Foundation. Use of published material is allowed under the following terms and conditions:

https://creativecommons.org/licenses/by-nc/4.0/legalcode. Copies of published material are allowed for personal or internal use. Sharing published material for non-commercial purposes is subject to the following conditions:

https://creativecommons.org/licenses/by-nc/4.0/legalcode, sect. 3. Reproducing and sharing published material for commercial purposes is not allowed without permission in writing from the publisher. 
with others, that Wnt/PCP components are expressed differently in CLL subgroups defined by IGHV mutational status and CLL aggressiveness. ${ }^{11-13}$ It has also been well described that deregulated ROR1 and Wnt/PCP pathway affect CLL cell migration, survival and chemotaxis. ${ }^{11,13,14}$ Despite the generally accepted importance of ROR1/PCP signaling in CLL, surprisingly little is known about downstream effectors and links to other signaling pathways critical for CLL pathogenesis.

In this study, we focused on the analysis of ROR1 downstream signaling in CLL. We took advantage of the proteomic approach and analyzed the protein composition of endogenous ROR1 complexes from primary CLL cells. This unbiased approach allowed us to identify a poorly known protein, Cordon-blue protein-like 1 (COBLL1), as a novel ROR1 binding partner. Examining COBLL1 expression in CLL cells showed that COBLL1 expression can serve as an independent molecular marker in U-CLL: U-CLL COBLL1-high patients had a deregulated response to microenvironmental stimuli and significantly worse prognosis, resulting in shorter overall survival (OS) and time to second treatment (TTST). These data further pinpoint the importance of the ROR1/PCP signaling axis in CLL and identify COBLL1 as an important and clinically relevant regulator of this process.

\section{Methods}

\section{Patients and samples}

All samples were taken after informed consent in accordance with the Declaration of Helsinki, under protocols approved by the Ethical Committee of the University Hospital Brno, Czech Republic.

Peripheral blood (PB) B cells from CLL patients or healthy volunteers and non-malignant tonsillar tissue were separated by nonB-cell depletion (RosetteSep CD3+ Cell Depletion Cocktail, RosetteSep B Cell Enrichment Cocktail, StemCell Technologies or magnetic B-cell isolation kit II, Miltenyi Biotec). Isolated B-cell purity was assessed by flow cytometry and exceeded $98 \%$. Tonsillar B cells were stained and sorted as described previously. ${ }^{11}$
RNA was extracted with TriReagent (Molecular Research Center). For information on how IGHV mutation status ${ }^{15}$ and genetic aberrations ${ }^{16-20}$ were determined and HEK293 and MAVER-1 cells cultured, see the Online Supplementary Appendix.

Mass spectrometry, transfection, immunoprecipitation, immunofluorescence and western blotting

To identify and confirm potential ROR1 binding partners, immunoprecipitation of ROR1 from primary CLL cells coupled to mass-spectrometry, ${ }^{21,22}$ transfection of HEK 293 cells, ${ }^{10,23}$ immunoprecipitation of MAVER-1 and transfected HEK293 cells, ${ }^{11}$ immunofluorescence of transfected HEK293 cells ${ }^{24}$ and western blotting ${ }^{25}$ were performed as previously described. For details, see the Online Supplementary Appendix.

\section{Gene expression analysis}

COBLL1 and ROR1 mRNA expression was assessed using qRTPCR. Three COBLL1 expression datasets were obtained; for details see the Online Supplementary Appendix. Since all datasets showed a similar bimodal distribution (Online Supplementary Figure S1), $\mathrm{dCt}$ values $\left(\mathrm{dCt}=\mathrm{Ct}_{\mathrm{COBLL1}}-\mathrm{Ct}_{\text {mean of reference genes }}\right)$ were normalized using the mean expression and standard deviation of the U-CLL samples and subsequently merged into one dataset. ROR1 mRNA expression was examined as previously described. ${ }^{13}$ The expression was further calculated from dCt values $(R O R 1)$ and normalized $\mathrm{dCt}$ values (dCtN, COBLL1) by the $2^{\mathrm{dct}} \times 100 \%$ and $2-$ ${ }^{\mathrm{dCAN}} \mathrm{x} 100 \%$ method, respectively.

\section{Transwell assay}

Cell migration in RPMI supplemented with 1\% FBS and antibiotics towards chemokines CCL19 or CXCL12 (200 ng/mL; 350NS-010, 361-MI-025, R\&D Systems) or chemokine-free media was analyzed as described previously. ${ }^{13}$ Migrated cells were counted using Accuri C6 flow cytometer (BD Biosciences).

\section{BCR stimulation}

The protocol previously described by Palomba et al. was adopted. ${ }^{26}$ For response quantification, phosphorylation increase was assessed and calculated as a ratio of positive cells in a stimulated and unstimulated sample. For details and western blot analysis, see the Online Supplementary Appendix.

Table 1. Results of the proteomic analysis of proteins co-immunoprecipitated with ROR1 from chronic lymphocytic leukemia samples.

\begin{tabular}{|c|c|c|c|c|c|c|}
\hline Accession $n$. & Protein name & $\# 1$ & \#2 & $\# 3$ & $\# 4$ & $\# 5$ \\
\hline ROR1_HUMAN & Tyrosine protein kinase transmembrane receptor & & & & & \\
\hline & ROR1 OS Homo sapiens GN ROR1 PE 2 SV 2 & $\mathrm{x}$ & $\mathrm{x}$ & $\mathrm{x}$ & $\mathrm{x}$ & $\mathrm{x}$ \\
\hline O75805_HUMAN & HOXA 9A OS Homo sapiens GN HOXA 9 PE 2 SV 1 & $\mathrm{x}$ & & & $\mathrm{x}$ & \\
\hline CENPE_HUMAN & $\begin{array}{l}\text { Centromere associated protein E OS Homo sapiens } \\
\text { GN CENPE PE } 1 \text { SV } 2\end{array}$ & & $\mathrm{x}$ & & & $\mathrm{x}$ \\
\hline COBL1_HUMAN & $\begin{array}{l}\text { Cordon bleu protein like } 1 \text { OS Homo sapiens } \\
\text { GN COBLL1 PE } 1 \text { SV } 2\end{array}$ & $\mathrm{x}$ & $\mathrm{x}$ & & & \\
\hline S17A4_HUMAN & $\begin{array}{l}\text { Putative small intestine sodium dependent phosphate } \\
\text { transport protein OS Homo sapiens GN SLC17A4 PE }\end{array}$ & $\mathrm{x}$ & & & $\mathrm{x}$ & \\
\hline APC2_HUMAN & $\begin{array}{l}\text { Adenomatous polyposis coli protein } 2 \text { OS Homo sapiens } \\
\text { GN APC2 PE } 1 \text { SV } 1\end{array}$ & & $\mathrm{x}$ & & & $\mathrm{x}$ \\
\hline KINH_HUMAN & $\begin{array}{l}\text { Kinesin } 1 \text { heavy chain OS Homo sapiens GN KIF5B } \\
\text { PE } 1 \text { SV } 1\end{array}$ & $\mathrm{x}$ & & & & $\mathrm{x}$ \\
\hline
\end{tabular}




\section{Statistical analysis and data visualization}

For statistical analysis, GraphPad Prism 5 (GraphPad Software), Statistica 10 (StatSoft) and R v.3.1.2.27 supplemented with a KEGG profile package ${ }^{28}$ were used. Genomic aberrations were visualized as Circos plots. ${ }^{29}$ COBLL1-linked signaling pathways were analyzed using CLLE-ES dataset (www.icgc.org). ${ }^{30}$ The cut off dividing patients into COBLL1-low and COBLL1-high subgroups was determined according to their OS. Kaplan-Meier curve dichotomization was accessed for each $\mathrm{dCtN}_{\mathrm{COBLL1}}$ and the value with the strongest difference was further used as cut off. For details, see the Online Supplementary Appendix.

\section{Results}

COBLL1 is a novel binding partner of ROR1

In order to investigate how ROR1 modulates CLL biology and pathogenesis, we decided to apply a proteomic approach and looked for novel ROR1 protein interaction partners. We immunoprecipitated endogenous ROR1 molecular complexes from the primary CLL cells of 5 CLI patients using anti-ROR1 specific antibody and analyzed the proteins pulled down with mass spectrometry. The hits that were identified in the ROR1 pulldown in at least
A

HEK293

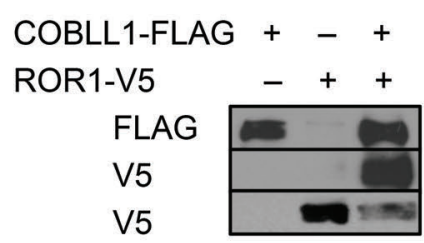

C

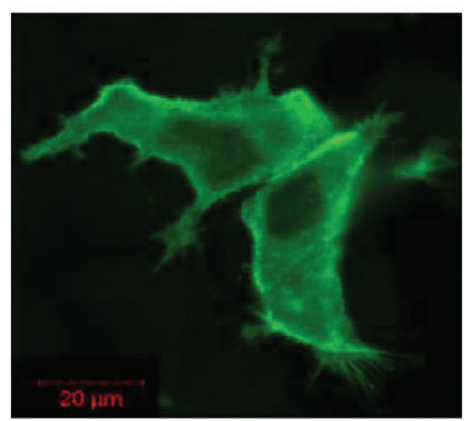

FLAG

D

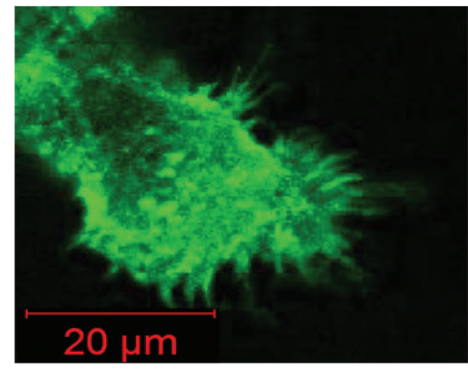

FLAG
MAVER-1
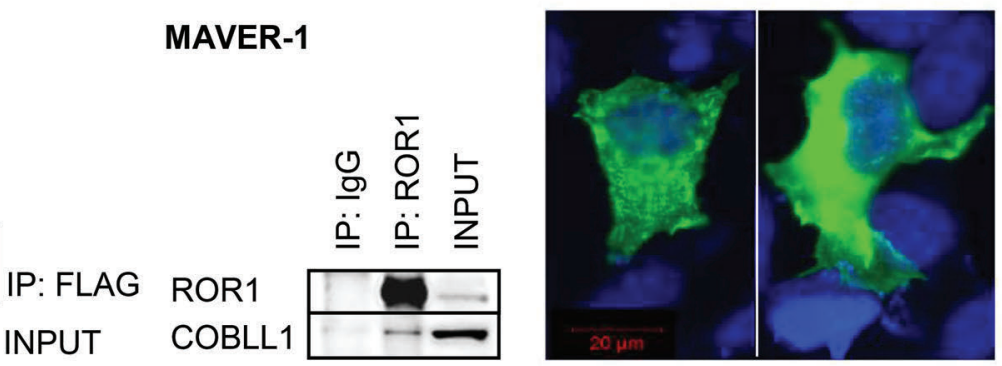

FLAG + nuclei

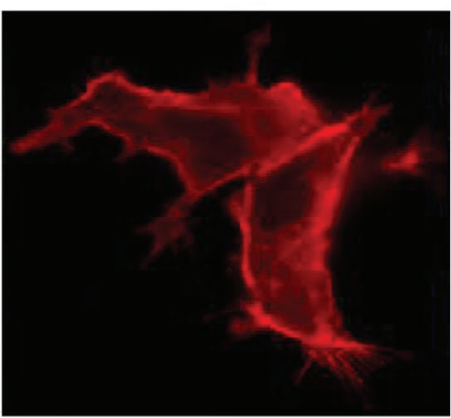

V5

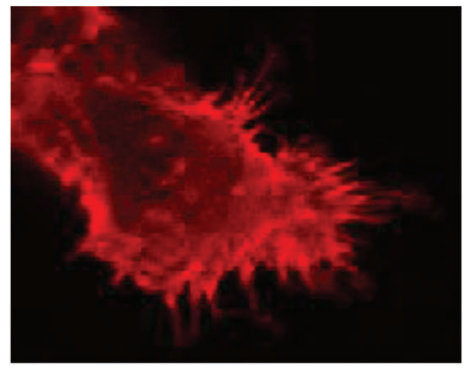

V5

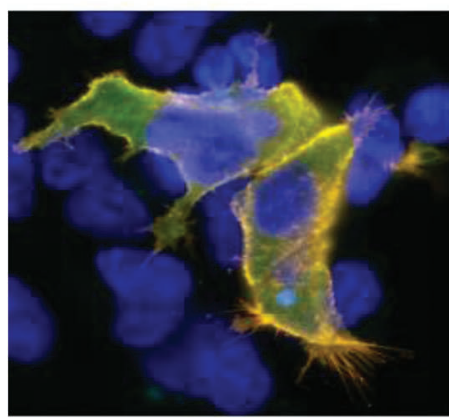

merge + nuclei

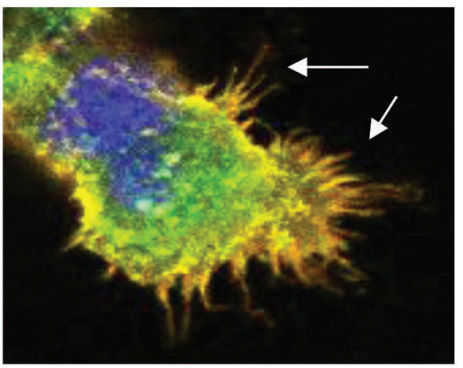

merge + nuclei

Figure 1. COBLL1 is an ROR1-interaction partner. (A) (Left) COBLL1-ROR1 complex was efficiently immunoprecipitated in HEK293 cells transfected with plasmids encoding FLAG-tagged COBLL1 and V5-tagged ROR1. (Right). Endogenous COBLL1 was pulled down with endogenous ROR1 in MAVER-1 cells; unspecific IgG was used as a negative control. Immunoprecipitation input is loaded on the right. Protein levels were determined using western blotting and anti-FLAG, anti-V5, anti-ROR1 and anti-COBLL1 antibodies. IP: immunoprecipitation. (B-D) Immunofluorescence of HEK293 cells transfected with plasmids encoding FLAG-COBLL1 (B-D) and V5ROR1 (C and D). COBLL1 over-expressed in HEK293 cells shows mostly cytoplasmic localization (B), but co-localizes with ROR1 in the membrane when ROR1 is coexpressed (C). The most efficient ROR1 and COBLL1 co-localization is observed in filopodia formed as a consequence of ROR1 overexpression (D, indicated by arrows). Protein expression was visualized using anti-FLAG, anti-V5 and corresponding secondary fluorescein-conjugated antibodies. Nuclei were visualized using DAPI staining. 
2 patients are shown in Table 1 . We compared this list of putative ROR1 interaction partners with the microarraybased dataset of genes differentially expressed in M-CLL versus U-CLL samples. ${ }^{31}$ This comparison pointed out the cordon blue protein-like 1 (COBLL1) protein as one of the most promising targets. In the next step, we focused on the validation and functional characterization of COBLL1, encoded by the COBLL1 gene.

First, we aimed to independently confirm that COBLL1 can indeed physically interact with ROR1. We transfected
HEK293 cells with plasmids encoding FLAG-tagged COBLL1 and V5-tagged ROR1 plasmids, and immunoprecipitated COBLL1 using anti-FLAG specific antibody. As shown in Figure 1A left, ROR1 can be efficiently coimmunoprecipitated with COBLL1. In order to confirm the interaction on an endogenous level in lymphoid cells, we also co-immunoprecipitated COBLL1 in endogenous ROR1 pulldown using anti-ROR1 antibody from protein lysates of MAVER-1 cells, ${ }^{32}$ a mantle cell lymphoma cell line expressing both COBLL1 and ROR1 (Figure 1A right).
A

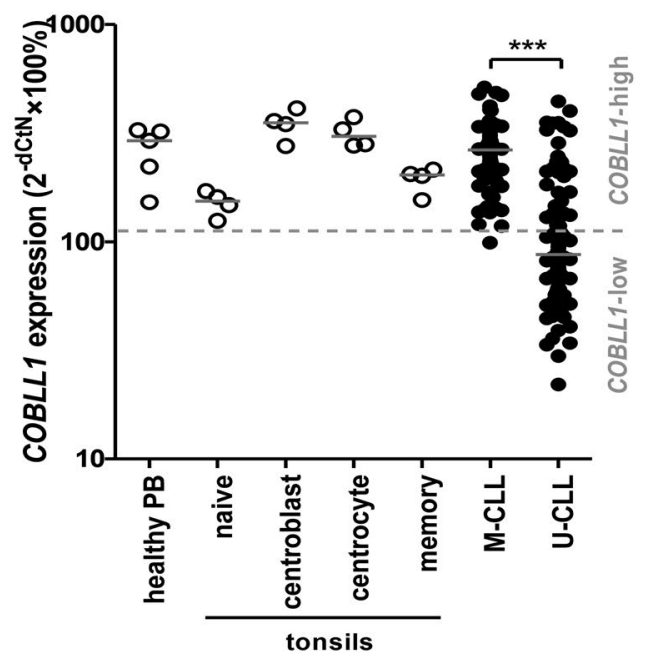

C

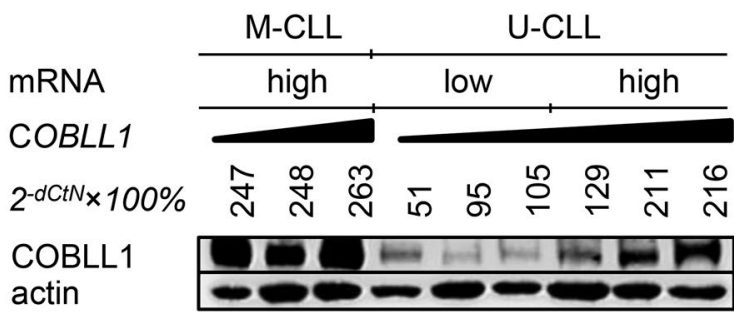

$\mathbf{E}$

\begin{tabular}{|c|c|c|c|c|c|c|c|c|}
\hline \multirow[b]{3}{*}{ Pationt } & \multicolumn{8}{|c|}{ U-CLL } \\
\hline & \multicolumn{4}{|c|}{ COBLL1-low } & \multicolumn{4}{|c|}{ COBLL1-high } \\
\hline & 1 & 1 & 2 & 2 & 3 & 3 & 4 & 4 \\
\hline Treatment & - & - & - & + & - & - & - & + \\
\hline OBLL1 & & & & & 6 & $=$ & $=$ & e \\
\hline
\end{tabular}

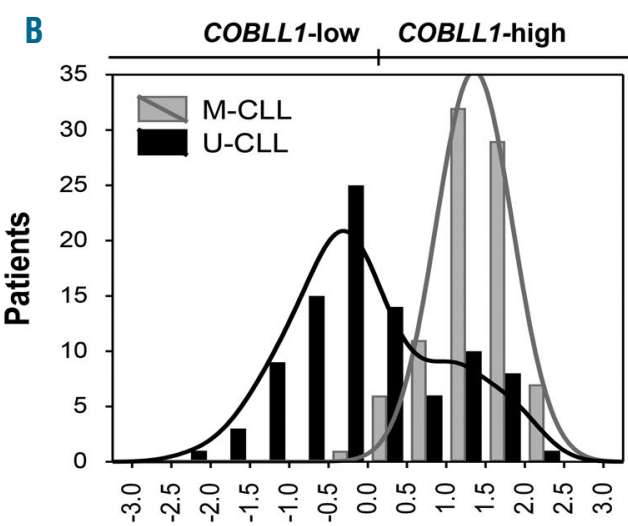

COBLL1 expression (-dCtN)

D

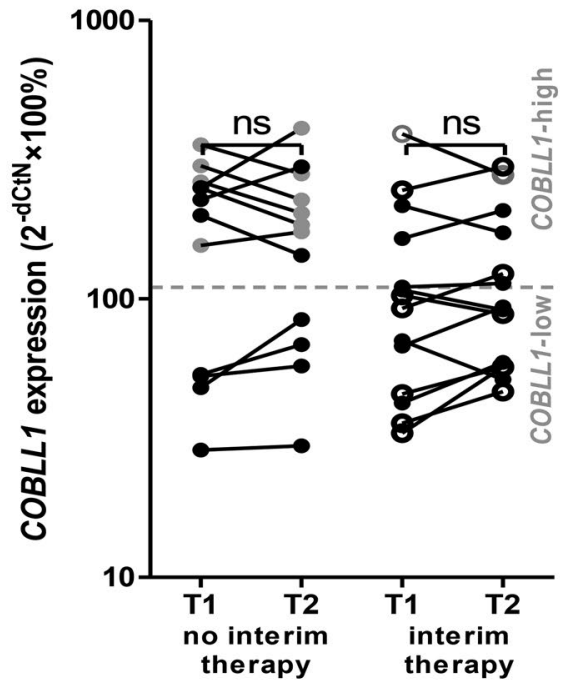

Figure 2. COBLL1 expression in chronic lymphocytic leukemia (CLL) and non-malignant B cells. (A) COBLL1 mRNA expression in 86 mutated CLL (M-CLL), 92 unmutated CLL (U-CLL), and healthy B cells isolated from 4 tonsils and 5 peripheral blood (PB) samples. Individual dots represent individual patients. Full lines indicate median. dCtN - dCt value normalized for three independent datasets (see Methods); $* * * P<0.0001$, Mann-Whitney test. (B) COBLL1 expression histogram follows a bimodal distribution pattern in U-CLL. (C) COBLL1 protein levels correspond very well with COBLL1 mRNA both in M-CLL and U-CLL cells. COBLL1 protein levels in CLL cells were determined using western blotting and anti-COBLL1 antibody. Actin was used as a loading control. Patient samples are ordered according to their IGHV mutation status and COBLL1 mRNA expression [in the ascending order; numbers indicate patients' relative COBLL1 expression determined by qRT-PCR (see A)]. (D) COBLL1 mRNA expression does not change with time or treatment. COBLL1 expression was analyzed in each patient at two time points (T1 and T2, connected by line) with (left; $6 \mathrm{M}$-CLL, $6 \mathrm{U}-\mathrm{CLL}$ ) or without (right; $1 \mathrm{M}$-CLL, $13 \mathrm{U}$-CLL) therapy in the interim. Patients were administrated mainly fludarabine-cyclophosphamide-rituximab (FCR) regimen. • M-CLL, • U-CLL: open circle; FCR; full circle: other therapy. Wilcoxon signed rank test. ns: not significant. (E) COBLL1 expression does not change with time or treatment. Protein expression was detected in 2 U-CLL COBLL1-low patients (1 and 2) and 2 U-CLL COBLL1-high patients (3 and 4) at two time points with or without therapy in the interim. Western blotting and anti-COBLL1 and anti-actin (as a loading control) antibody was used. 
These co-immunoprecipitation experiments confirmed that ROR1 can indeed interact with COBLL1 both at the exogenous as well as endogenous level.

ROR1 is a transmembrane receptor, which in most cell types has the capability to induce filopodia formation. ${ }^{33}$ Immunofluorescence staining of transfected HEK293 cells showed that, when solely COBLL1 is expressed, it was localized mainly in the cytoplasm (Figure 1B). However, when ROR1 was co-expressed, the COBLL1 signal was detected predominantly in the plasma membrane (Figure
1C) where it co-localized with ROR1. The co-localization was most prominent in the filopodia, which formed as a consequence of ROR1 overexpression (Figure 1D). These data demonstrate that COBLL1 is a true ROR1 binding partner which is recruited to the ROR1 signaling complexes in the membrane.

COBLL1 expression levels vary dramatically among CLL

To evaluate COBLL1 relevance in CLL, we analyzed its expression in the cohort of 178 CLL untreated patients (86

A
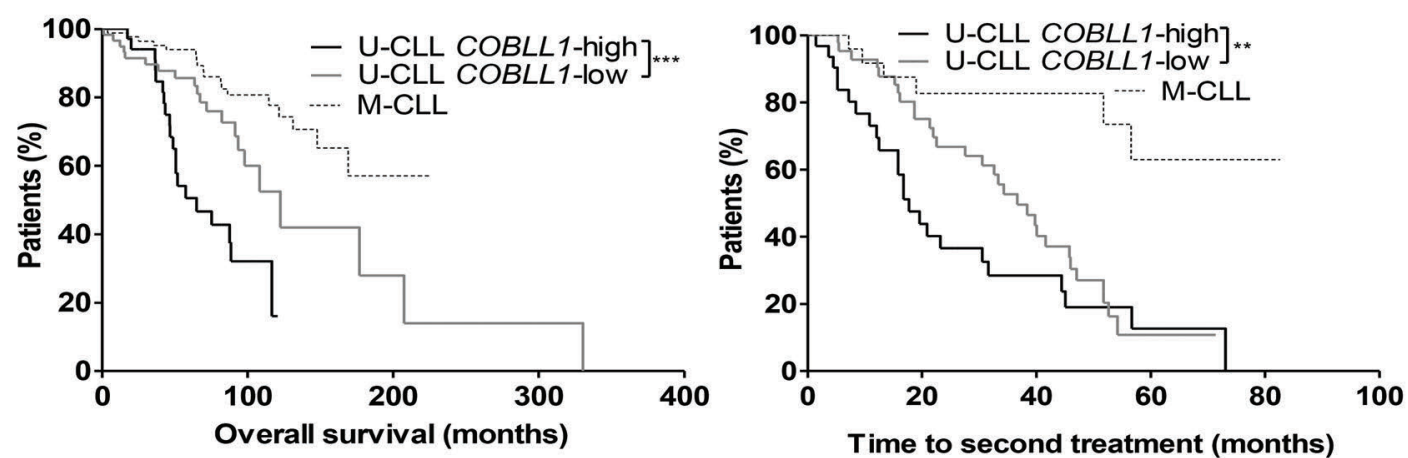

B<smiles>[CaH][GeH3]</smiles>

U-CLL U-CLL
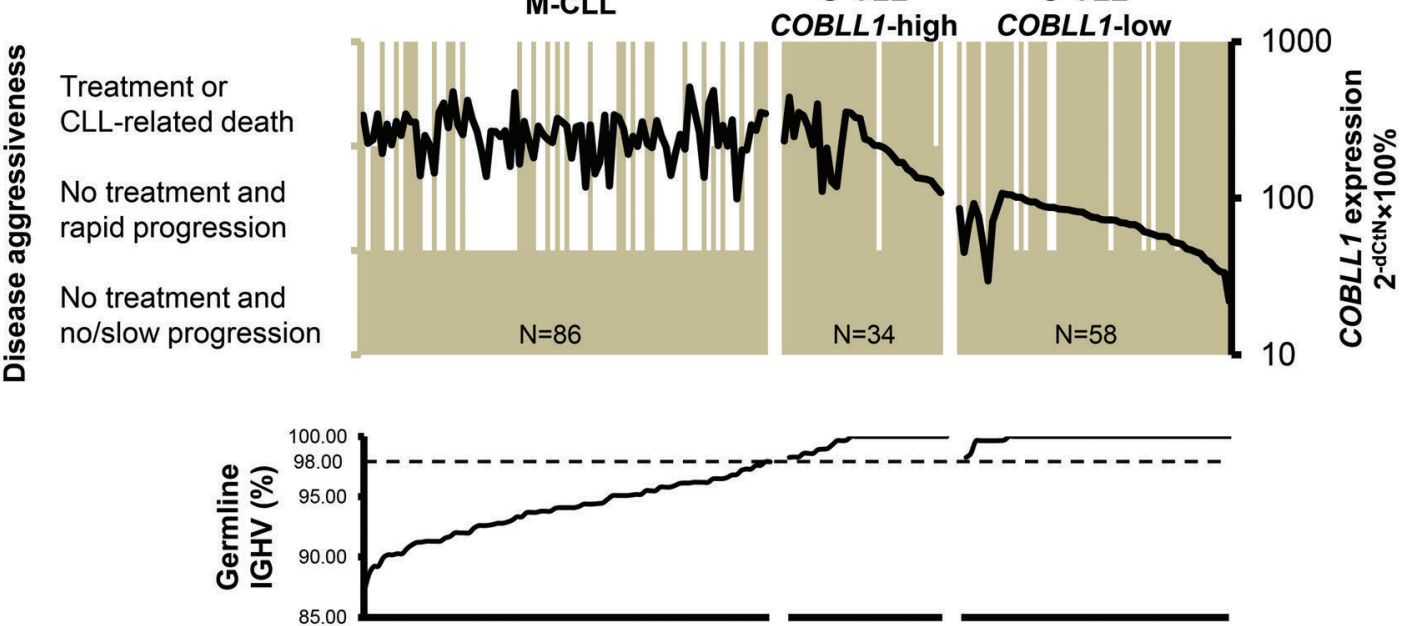

Patients

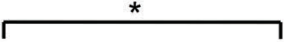

\begin{tabular}{lccc}
\hline Patients & M-CLL (\%) & U-CLL & U-CLL \\
\hline Treatment or CLL-related death & 31.4 & 94.1 & COBLL1-high (\%) \\
No treatment and rapid progression & 8.1 & 2.9 & 0.0 \\
No treatment and no/slow progression & 60.5 & 2.9 & 17.2 \\
\hline
\end{tabular}

Figure 3. Unmutated chronic lymphocytic leukemia (U-CLL) COBLL1-high patients show significantly shorter survival and progress more often compared to U-CLL COBLL1-low patients. (A) U-CLL COBLL1-high patients show shorter overall survival (left) and time to second treatment (right). Survival data are presented using Kaplan-Meier plots and tested by Gehan-Breslow-Wilcoxon test. (B) U-CLL COBLL1-high patients progress more often than patients in other groups. Progression (left y-axis, gray columns) categorized as 1 - no treatment and no/slow progression (clinical stage Rai 0/I at both diagnosis and sampling); 2 - no treatment but rapid progression (clinical stage Rai 0/I at diagnosis and II/III/IV at sampling); 3 - treatment or CLL-related death (various clinical stages at diagnosis and sampling). Patients are grouped based on their IGHV mutation/COBLL1 expression status, and ordered according to germline IGHV (in the ascending order, X-axis) and COBLL1 expression (descending order, full line, right y-axis). (A left and B). N: 86 mutated CLL (M-CLL), 58 U-CLL COBLL1-low, 34 U-CLL COBLL1-high. (A right). N: 28 M-CLL, 48 U-

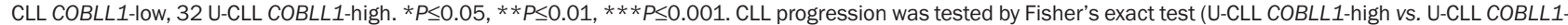
low). 
Table 2. Multivariate Cox analysis in unmutated chronic lymphocytic leukemia overall survival.

\begin{tabular}{|c|c|c|c|c|}
\hline & \multirow[t]{2}{*}{ HR } & \multicolumn{2}{|c|}{ 95\%Cl for HR } & \multirow[t]{2}{*}{$P$} \\
\hline & & Lower & Upper & \\
\hline COBLL1 - high & 2.924 & 1.372 & 6.232 & 0.005 \\
\hline Age at diagnosis & 1.025 & 0.982 & 1.070 & 0.261 \\
\hline \multicolumn{5}{|c|}{ Risk according to Rai stage at diagnosis* } \\
\hline Intermediate & 1.514 & 0.668 & 3.427 & 0.320 \\
\hline High & 6.029 & 2.003 & 18.145 & 0.001 \\
\hline CD38 - positive & 3.086 & 1.087 & 8.757 & 0.034 \\
\hline \multicolumn{5}{|c|}{ Cytogenetic hierarchical model ${ }^{* *}$} \\
\hline $\operatorname{del}(17 p)$ & 5.049 & 1.691 & 15.076 & 0.004 \\
\hline $\operatorname{del}(11 q)$ & 2.503 & 0.853 & 7.344 & 0.095 \\
\hline trisomy 12 & 0.816 & 0.192 & 3.468 & 0.783 \\
\hline $\operatorname{del}(13 q)$ & 1.232 & 0.399 & 3.811 & 0.717 \\
\hline
\end{tabular}

${ }^{*}$ Compared to low risk. ${ }^{*}$ Compared to normal karyotype. HR: hazard ratio; CI: confidence interval. Statistically significant $P$-values are highlighted in bold.

M-CLL, 92 U-CLL) and compared it with non-malignant B cells from PB (5 samples) and tonsillar tissue (4 samples). COBLL1 was highly expressed in normal $\mathrm{PB}$ and tonsillar tissue (Figure 2A). The expression in individual tonsillar Bcell subpopulations varied; COBLL1 expression in centroblasts and centrocytes was increased compared to naive and memory $B$ cells.

The expression in CLL cells differed significantly according to the IGHV mutation status $(P<0.0001$, MannWhitney test). COBLL1 levels were higher in M-CLL patients with an expression comparable to that of healthy tonsillar and PB B cells. On the contrary, the COBLL1 expression in U-CLL showed bimodal distribution (Figure 2B). A subgroup of U-CLL patients expressed COBLL1 at a level comparable with M-CLL patients, but in the majority of U-CLL samples COBLL1 expression was much lower. Since the COBLL1 expression had such a clearly bimodal distribution in all three independently analyzed datasets (see Methods section and Online Supplementary Figure S1), we set a cut off to distinguish COBLL1-high and COBLL1-low patients (for details see Methods section). The cut off is set close to the local distribution minimum (Figure 2B). Following this approach, all but one $\mathrm{M}$ CLL patient was classified as COBLL1-high. The majority of U-CLL patients $(n=58 ; 63 \%)$ were classified as COBLL1-low, whereas the remaining U-CLL patients ( $\mathrm{n}=34 ; 37 \%$ ) were classified as COBLL1-high. Different expression in both cohorts was also confirmed at protein level (Figure 2C).

To analyze the changes in COBLL1 expression over time and after treatment, we examined 26 patients at two time points (7 M-CLL, $19 \mathrm{U}$-CLL) (Figure 2D). A part of the cohort was not treated in the interim (6 M-CLL, $6 \mathrm{U}$ CLL; median 37 months), whereas the remaining patients (1 M-CLL, 13 U-CLL; median 35 months) were administrated a fludarabine-cyclophosphamide-rituximab regimen or another chemoimmunotherapy. The COBLL1 expression category did not change, with one exception: COBLL1 expression was slightly increased after treatment in one borderline U-CLL COBLL1-low patient. We also examined the changes in expression at protein level in 4 U-CLL patients and obtained similar data (2 U-CLL COBLL1-low, 2 U-CLL COBLL1-high; 2 with treatment in the interim, 2 without treatment in the interim) (Figure 2E). This suggests that COBLL1 expression at mRNA as well as protein level does not dramatically change with time or treatment.

Recently, CLL patients with high ROR1 expression were found to suffer from a more aggressive disease. ${ }^{34}$ Since COBLL1 and ROR1 form a protein complex, we correlated COBLL1 and ROR1 expression (protein levels of COBLL1 and ROR $1^{11}$ correspond well with mRNA levels) (Figure 2C) but did not find any correlation (Online Supplementary Figure S2A). We were also unable to detect any obvious changes in COBLL1 levels or phosphorylation (detected as phosphorylation-dependent mobility shift) upon activation of ROR1 by its ligand Wnt-5a (Online Supplementary Figure S2B). This suggests that COBLL1 rather represents an independently-regulated ROR1 signaling modulator than a bona fide component of ROR1 signaling pathway.

\section{High COBLL1 expression identifies a subgroup of U-CLL patients with inferior prognosis independent of other prognostic markers}

To explore the possible COBLL1 association with CLL disease course, we analyzed the survival of M-CLL, UCLL COBLL1-low and U-CLL COBLL1-high patients. As expected, M-CLL patients showed the best prognosis according to $O S$ and time to second treatment (median OS and TTST not reached; M-CLL vs. U-CLL COBLL1low $P_{O S}=0.0389, P_{T T S T}=0.0104 ; \mathrm{M}-\mathrm{CLL}$ vs. U-CLL COBLL1-high $P_{O S}<0.0001, P_{T T S T}=0.0004$, Gehan-BreslowWilcoxon test) (Figure $3 \mathrm{~A}$ ). The survival of U-CLL patients differed according to COBLL1 expression. U-CLL COBLL1-high patients showed a more aggressive disease course (median OS 65 months, TTST 17 months), whereas the U-CLL COBLL1-low patients progressed more slowly (median OS 123 months, TTST 37 months; U-CLL COBLL1-high vs. U-CLL COBLL1-low $P_{O S}=0.0086$, $\left.P_{\text {TTST }}=0.0116\right)$. There was no significant difference in time to first treatment (TTFT) between U-CLL COBLL1-high and low (Online Supplementary Figure S3).

To get further insight into the role of COBLL1, we categorized M-CLL, U-CLL COBLL1-low and U-CLL COBLL1-high patients, based on the aggressiveness of the 
disease. This parameter was defined based on the disease behavior between diagnosis and sampling (median time between diagnosis and sampling: 35 months in M-CLL, 11 months in U-CLL COBLL1-high, 19 months in U-CLL COBLL1-low). Patients were categorized into three groups: 1) no treatment and no/slow progression (clinical stage Rai 0/I at sampling); 2) no treatment but rapid progression (progression into clinical stage Rai II/III/IV at sampling); 3) treatment or CLL-related death. U-CLL
COBLL1-high progressed more often than U-CLL COBLL1-low ( $P=0.0297$, Fisher's exact test) (Figure 3B). UCLL COBLL1-high progressed in almost all cases; only $3 \%$ did not progress versus $17 \%$ in U-CLL COBLL1-low patients. In line with this observation, treatment or CLLrelated death occurred more often in the U-CLL COBLL1high patients than in U-CLL COBLL1-low patients $(94 \%$ vs. $83 \%$ ).

To further confirm the difference in U-CLL patients

A

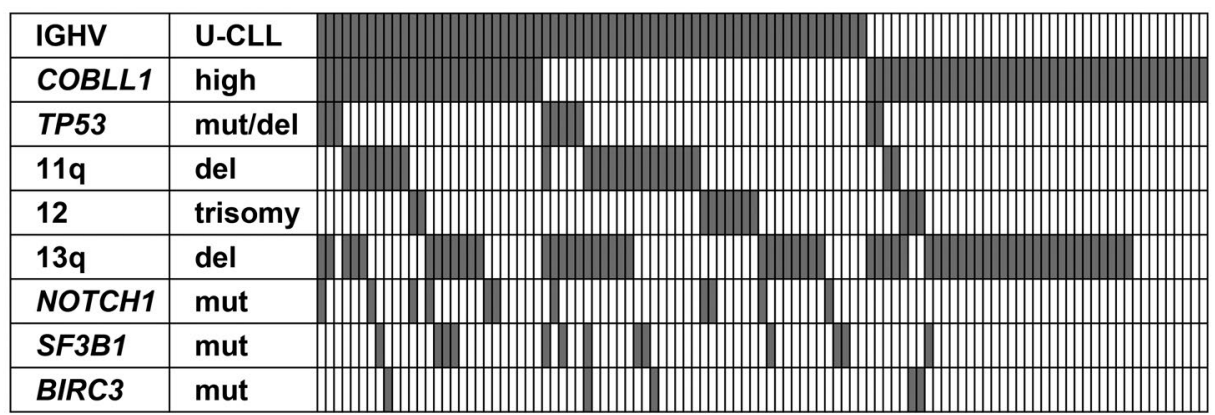

B

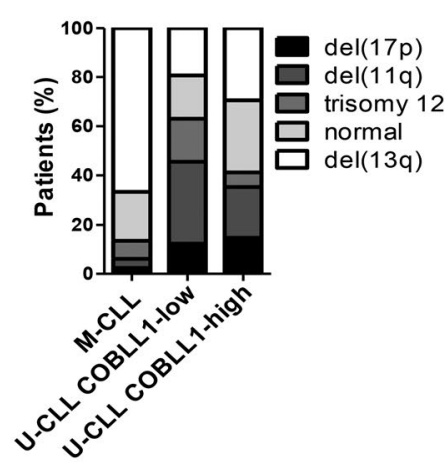

C

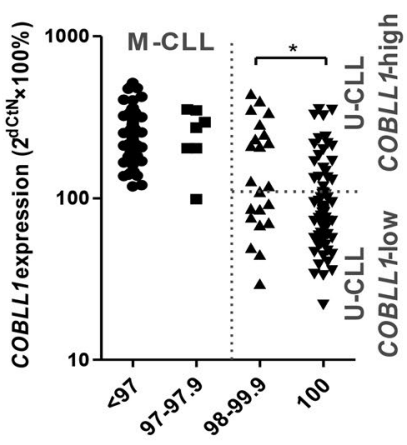

Germline IGHV (\%)
D

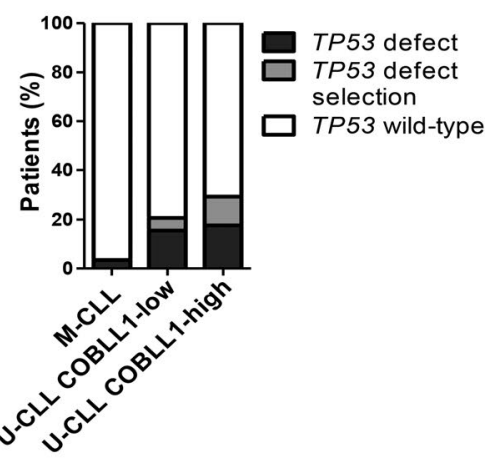

$\mathbf{E}$
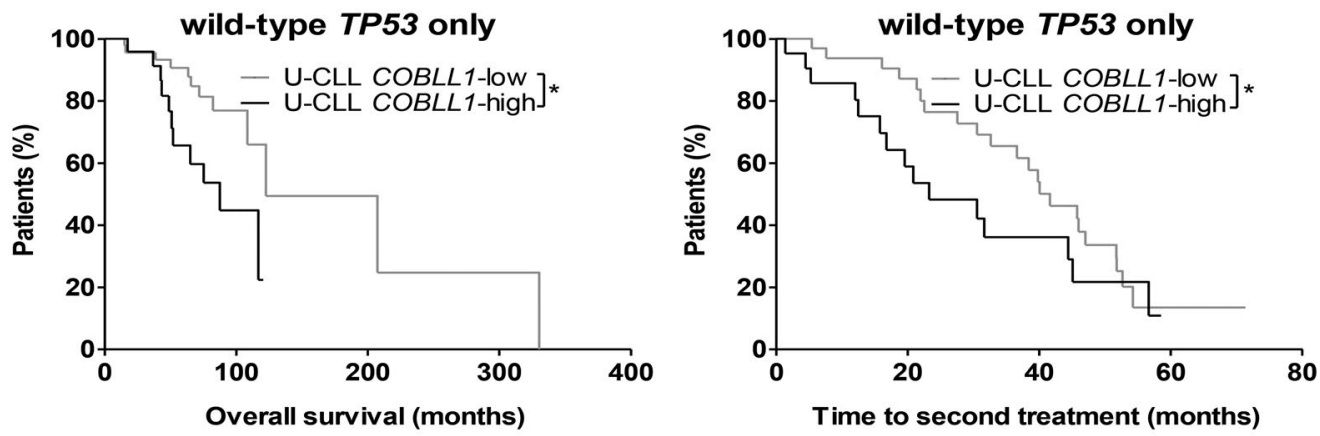

Figure 4. The survival difference between unmutated chronic lymphocytic leukemia (U-CLL) COBLL1-high and U-CLL COBLL1-low is not caused by recurrent mutations and chromosomal abnormalities. (A-D) Samples were analyzed by I-FISH [del(17p), del(11q), trisomy 12, del(11q)] and sequencing (mutations in IGHV, TP53, BIRC3, NOTCH1, SF3B1). TP53 defect - TP53 mutation, deletion or both. (A) U-CLL COBLL1-low and U-CLL COBLL1-high patients do not exhibit any differences in the occurrence of recurrent defects [TP53 defect, BIRC3, NOTCH1 and SF3B1 mutations, del(11q), trisomy 12, del(13q)] or (B) in cytogenetic aberrations evaluated according to the hierarchical model. ${ }^{36}$ (C) Expression of COBLL1 categorized according to the IGHV mutation load. (D) U-CLL COBLL1-high patients exhibit non-significantly higher incidence of TP53 defect at diagnosis or its later selection. (A) 41 mutated CLL (M-CLL), 37 U-CLL COBLL1-low, 29 U-CLL COBLL1-high. (B-D) 86 MCLL, 58 U-CLL COBLL1-low, 34 U-CLL COBLL1-high. (D) Adverse survival of U-CLL COBLL1-high patients is retained even in TP53 wild-type patients. (D) (Left) Overall survival: 46 U-CLL COBLL1-low, 24 U-CLL COBLL1-high. (D) (Right) Time to second treatment: 37 U-CLL COBLL1-low, 22 U-CLL COBLL1-high. *P $\leq 0.05, * * P \leq 0.01$, $* * * P \leq 0.001$. Aberrations frequency tested by Fisher's exact test, survival data tested by Gehan-Breslow-Wilcoxon test, germline IGHV tested by Mann-Whitney test. 
overall survival, we analyzed our patient cohorts separately (cohort B vs. cohort $\mathrm{A}+\mathrm{C}$ ). The U-CLL COBLL1-high patients showed a shorter OS than U-CLL COBLL1-low in both cases (58 vs. 75 months in cohort B and 75 vs. 123 months in cohort $\mathrm{A}+\mathrm{C})$ (Online Supplementary Figure S4) but the difference was significant only in cohort $B$ $(P=0.0314$, Gehan-Breslow-Wilcoxon test); this is likely due to the relatively small number of patients.

The striking difference in survival of U-CLL COBLL1-

A

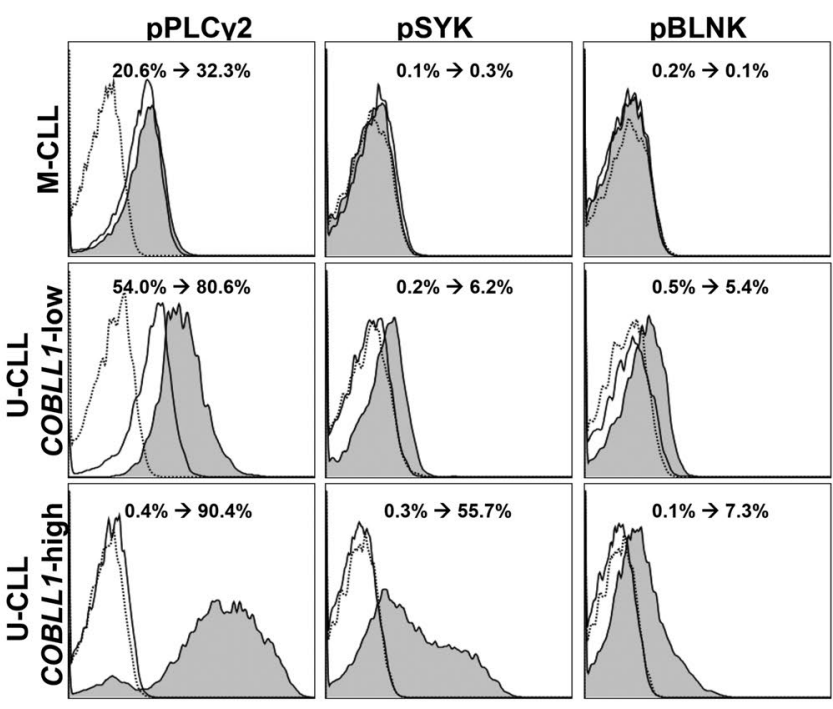

B

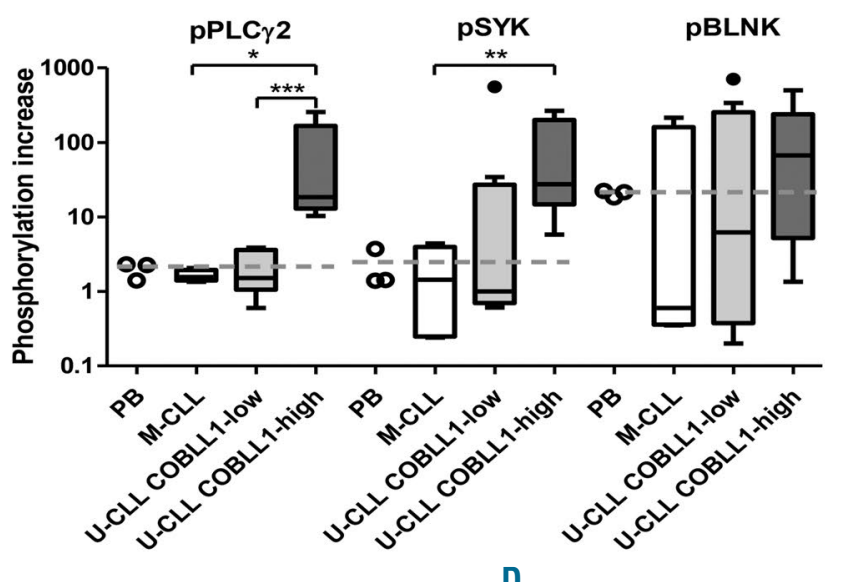

C

U-CLL $\overline{\text { COBLL1-high COBLL1-low }}$

anti-IgM

pY1217-PLCy2

pY525/526 -SYK

pS473-AKT

pT202/Y204-ERK1/2

loading control
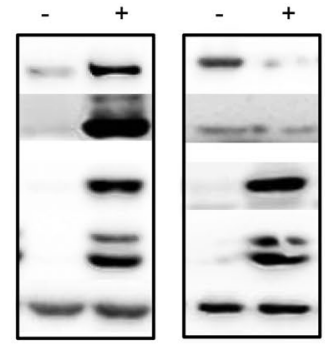

\begin{tabular}{|l|l|l|l|}
\hline $\begin{array}{l}\text { Spearman } \\
\text { correlation } \\
(P \text {-value })\end{array}$ & pSYK & pAKT & pERK1/2 \\
\hline pPLCy2 & $\mathbf{0 . 0 0 7 6}$ & 0.7330 & 0.2504 \\
\hline pSYK & - & $>0.9999$ & 0.3863 \\
\hline pAKT & $>0.9999$ & - & 0.2632 \\
\hline
\end{tabular}

Figure 5. U-CLL COBLL1-high cells show higher response upon BCR stimulation. (A and B). Chronic lymphocytic leukemia (CLL) cells (4 mutated CLL (M-CLL), 8 UCLL COBLL1-low, 6 U-CLL COBLL1-high) were stimulated for 4 minutes with anti-lgM and response to BCR stimulation was analyzed using phospho-specific antibodies targeted against pPLCy2, pSYK and pBLNK. (A) Representative examples of M-CLL, U-CLL COBLL1-low and U-CLL COBLL1-high patients. Histograms show a negative control (unstimulated non-stained sample, dotted line), unstimulated sample (full line) and IgM-stimulated sample (full line, gray area). Percentage of positive cells is indicated (unstimulated sample $\rightarrow$ stimulated sample). (B) Quantification of changes in the pPLCY2, pSYK and pBLNK. Phosphorylation increase (y-axis) was calculated as a ratio of positive cells in IgM-stimulated versus unstimulated samples. Box-and-Whisker plots show quartiles and median. Dashed line indicates phos-

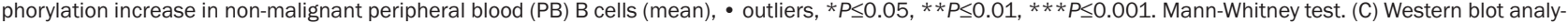
sis of representative U-CLL samples treated with anti-lgM and analyzed for activation of BCR components using phospho-specific antibodies - PLCY2 (pY1217), pSYK (pY525/526), pAKT (pS473) and pERK1/2 (pT202/Y204). Loading control: $\beta$-actin (left), total PLCy2 (right). (D) Correlation of the response at the level of individual kinases (Spearman correlation). Statistically significant $P$-values are highlighted in bold with gray background. See Online Supplementary Figure S7 for details and raw data. 
high and U-CLL COBLL1-low patients leads to compare the genetic aberrations which could influence the patients' prognosis in both cohorts. We analyzed cytogenetic aberrations $[\operatorname{del}(17 \mathrm{p}), \operatorname{del}(11 \mathrm{q})$, trisomy $12, \operatorname{del}(13 \mathrm{q})]$ and recurrent mutations (TP53, NOTCH1, BIRC3, SF3B1) in 107 patients (41 M-CLL, 37 U-CLL COBLL1-low, 29 U-CLL COBLL1-high) where all these data were available. We were unable to find any significant difference in U-CLL COBLL1-high versus COBLL1-low categories (see Figure 4A for brick plot, Online Supplementary Figure S5 for Circos plot, Figure $4 \mathrm{~B}$ for hierarchically categorized cytogenetic aberrations; ${ }^{35}$ Fisher's exact test).

Since U-CLL patients with borderline IGHV mutations have been shown to have a better prognosis than patients with truly unmutated IGHV, ${ }^{36}$ we also compared mutation load in U-CLL patients. The worse prognosis of U-CLL COBLL1-high patients could not be explained by difference in mutation load; on the contrary, borderline mutated patients (98-99.9\%) showed higher expression of COBLL1 than patients with $100 \%$ identity $(P=0.0219$, MannWhitney test) (Figure 4C).

We further assessed the influence of TP53 aberrations (mutations, deletions or both) present either before treatment or evolving during disease progression. Although UCLL COBLL1-high patients harbored TP53 aberrations more often and also lost wild-type TP53 more often during disease evolution, the differences were not significant $(P=0.4823$; Fisher's exact test) (Figure 4D). Furthermore, when we compared only wild-type TP53 patient survival, the U-CLL COBLL1-high patients still retained a worse OS and TTST (U-CLL COBLL1-low: median OS 122 months, median TTST 42 months; U-CLL COBLL1-high: median OS 88 months, median TTST 23 months; $P_{\text {OS }}=0.0276, P_{\text {TTST }}=0.0404$; Gehan-Breslow-Wilcoxon test) (Figure 4E).

To evaluate COBLL1 significance in U-CLL survival, we performed univariate and multivariate Cox regression analyses. The univariate analysis revealed COBLL1 status, age at diagnosis, Rai stage at diagnosis, CD38 expression and cytogenetic aberrations as significant prognostic factors for OS in U-CLL. Multivariate Cox regression analysis confirmed COBLL1 as an independent molecular marker (Table 2). In multivariate Cox regression analysis for TTST, COBLL1 did not retain independence.

Moreover, we did not find any difference in the clinical parameters such as leukocytosis, clinical stage, age or sex, explaining the short survival of U-CLL COBLL1-high patients. Due to the striking difference in TTST, we also investigated the administered treatment in detail (Online Supplementary Figure S6). We did not find any difference in patient treatment response, length or number of received treatment cycles or if categorized as full, reduced, interrupted or reduced therapy. Therefore, we assumed that the aggressive course of U-CLL COBLL1-high patients cannot be explained by any common unfavorable clinicobiological disease characteristics.

\section{U-CLL COBLL1-high cells show higher phosphorylation upon BCR stimulation}

To understand our findings in context, we performed detailed bioinformatics analysis of publicly available RNA sequencing data of $44 \mathrm{U}-\mathrm{CLL}$ samples ${ }^{30}$ (see Methods section). A subset of 1240 significantly COBLL1-correlated genes $(P<0.05$; Spearman test) (Online Supplementary Table S1) was selected for KEGG pathway analyses. Among the transcripts positively correlating with COBLL1 in U-CLL the genes associated with various cancer-linked signaling pathways and metabolic processes, including the B-cell receptor (BCR) pathway, were enriched (Online Supplementary Table S2). Progressive phosphorylation of BCR pathway components promotes cell survival, differentiation and proliferation in CLL (for review see ten Hacken and Burger ${ }^{37}$ ). Given the crucial biological and clinical importance of BCR signaling in CLL cells, we hypothesized that U-CLL COBLL1-high patients might have a deregulated response to BCR stimulation.

To investigate how U-CLL COBLL1-high cells respond to BCR stimulation, we adopted a previously described
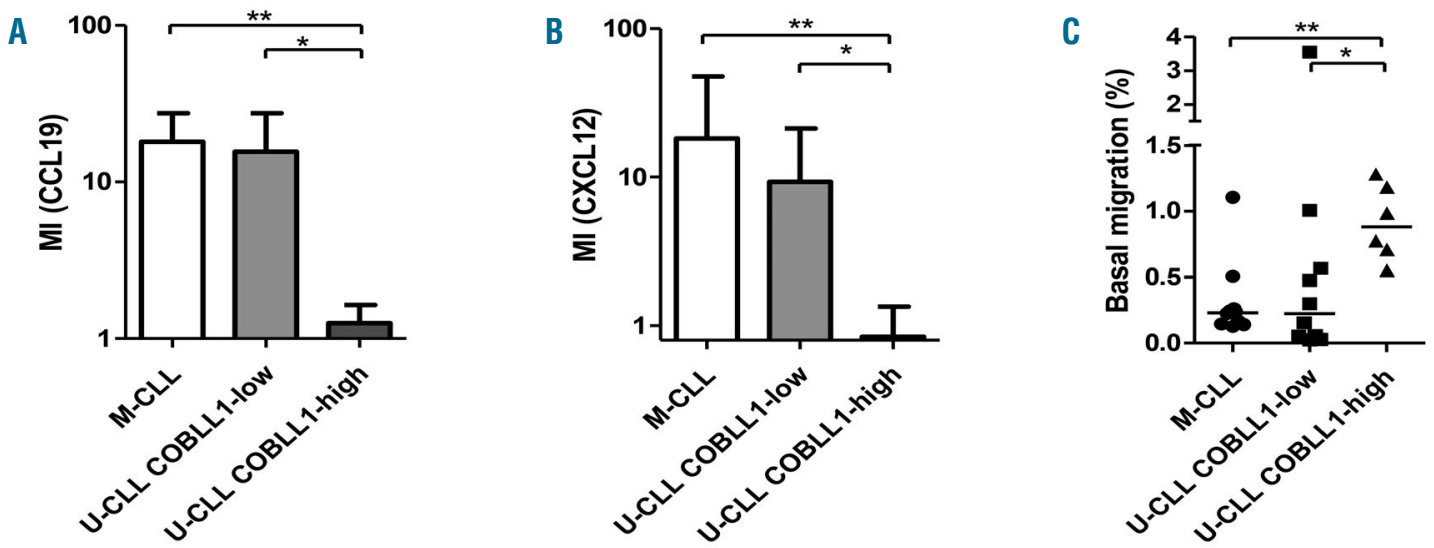

Figure 6. Unmutated chronic lymphocytic leukemia (U-CLL) COBLL1-high cells show deregulated chemotaxis and motility. Migratory properties of 10 mutated CLL (M-CLL), $10 \mathrm{U}-\mathrm{CLL}$ COBLL1-low, and 6 U-CLL COBLL1-high samples were assessed using transwell plates. (A) Chemotaxis towards chemokine CCL19 expressed as migration index (MI). (B) Chemotaxis towards chemokine CXCL12 expressed as MI. (C) Basal migration. MI was calculated as the number of cells migrated towards chemokine divided by the number of cells migrated in chemokine-free media. Basal migration was calculated as the percentage of migrated cells from all seeded cells. Each measurement was performed in a technical triplicate. Bars represent mean+Standard Deviation (S.D.) (A and B) Individual dots represent individual

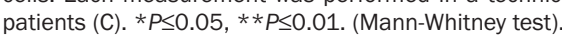


protocol, ${ }^{26}$ stimulated CLL cells and examined the phosphorylation level of selected BCR signaling pathway components (pPLC 2 , pSYK and pBLNK) via flow cytometry. Eighteen CLL samples (4 M-CLL, 8 U-CLL COBLL1-low, 6 U-CLL COBLL1-high) and 3 peripheral blood (PB) B-cell samples from healthy donors were analyzed. The response to anti-IgM was evaluated as a difference (fold change) in the number of positive cells in stimulated and unstimulated samples.

Not surprisingly, the response to BCR stimulation in CLL cells from individual patients was rather heterogeneous but still showed clear trends in the individual groups (see representative examples in Figure $5 \mathrm{~A}$ ). When quantified (Figure 5B), the number of pPLC $\gamma 2$-positive cells after BCR stimulation was dramatically increased only in U-CLL COBLL1-high [U-CLL COBLL1-high vs. UCLL COBLL1-low $(P=0.0007)$, U-CLL COBLL1-high vs. M-CLL $(P=0.0007)$, vs. M-CLL $(P=0.0139)]$. A similar trend could also be seen for pSYK [U-CLL COBLL1-high vs. UCLL COBLL1-low $(P=0.0609)$, U-CLL COBLL1-high vs. M-CLL $(P=0.0095)$, Mann-Whitney test] and pBLNK where U-CLL COBLL1-high cells responded best, albeit not with statistical significance. The non-malignant $\mathrm{PB}$ B-cell controls showed a uniform response, which was very similar to that of M-CLL (Figure $5 \mathrm{~B}$ ).

Interestingly, the western blot analysis confirmed in principle the differences in the activation of upstream BCR signaling components, namely PLC $\gamma 2$ and SYK, but we were unable to detect any differences between the groups at the activated AKT and ERK1/2 level (Figure 5C). Quantitative analysis of western blot data from a larger cohort of CLL samples ( $\mathrm{n}=10$ for $\mathrm{pAKT}, 11$ for all others) showed that pPLC $\gamma 2$ and pSYK signals correlated strongly with each other (Figure 5D, graphs in Online Supplementary Figure S7) but not with the pAKT and pERK1/2 signals that were almost uniformly induced in all patients (Online Supplementary Figure S7A). This suggests that regulating the upstream (PLC $\gamma 2$, SYK) and downstream (ERK1/2, AKT) BCR pathway module can differ. We conclude that U-CLL COBLL1-high patients exhibit an enhanced response to BCR stimulation, in particular at the level of upstream components such as pPLC $\gamma 2$ and pSYK.

\section{U-CLL COBLL1-high cells exhibit impaired migration and chemotaxis}

COBLL1 can physically interact with ROR1 and thus represents a candidate regulator of the non-canonical Wnt/PCP pathway. Since the Wnt/PCP pathway was shown to be involved in the migration of CLL cells, ${ }^{11,13}$ we analyzed their ability to respond to chemokines CCL19 and CXCL12, known to stimulate the cells via CCR7 and CXCR4 receptors, respectively. ${ }^{38}$ CLL cells were stratified according to the expression of COBLL1 and IGHV mutational status (10 M-CLL, $10 \mathrm{U}$-CLL COBLL1-low, 6 U-CLL COBLL1-high).

Generally, the chemotactic and migratory abilities differed according to the combination of IGHV status and COBLL1 expression (Figure 6). U-CLL COBLL1-high cells showed impaired chemotaxis towards chemokines CCL19 and CXCL12 and increased basal migration compared to U-CLL COBLL1-low and M-CLL cells [both CCL19 and CXCL12: U-CLL COBLL1-high vs. M-CLL $(P=0.0017)$, U-CLL COBLL1-high vs. U-CLL COBLL1-low $(P=0.0302)$; basal migration: U-CLL COBLL1-high vs. MCLL $(P=0.0030)$, U-CLL COBLL1-high vs. U-CLL COBLL1- low $(P=0.0420)$ Mann-Whitney test]. U-CLL COBLL1-low exhibited an intermediate response to chemokine stimuli. The deregulated migratory abilities of U-CLL COBLL1high cells further point out their altered microenvironmental interactions.

\section{Discussion}

In this study, we have identified COBLL1 as a novel binding partner for ROR1 in CLL. COBLL1 is an evolutionary conserved but very little known protein. Its mouse ortholog Cordon blue (Cobl) interacts with Vang-like protein 2 (Vangl2; a component of the Wnt/PCP pathway) and is required for neural tube closure, which is a process typically regulated by the Wnt/PCP pathway. ${ }^{39}$ The combination of these results and our findings suggests that COBLL1 can represent a Wnt/PCP pathway regulator in mammals. COBLL1 levels in CLL dramatically vary and do not correlate with ROR1. This opens up the possibility that the way COBLL1 affects ROR1 function may differ depending on the level of COBLL1.

COBLL1 links to human pathological conditions are very limited and restricted to the observation that COBLL1 upregulation is associated with a better prognosis after surgery in malignant pleural mesothelioma, where it acts as a negative regulator of apoptosis. ${ }^{40} \mathrm{On}$ the contrary, COBLL1 upregulation in chronic myeloid leukemia patients was recently associated with a reduction in nilotinib-dependent apoptosis, disease progression and shorter OS. ${ }^{41}$ Besides mature B cells, COBLL1 expression is detectable in various other cell types including other blood elements (such as T cells), although usually at a much lower level. ${ }^{42}$ Comparable or higher COBLL1 levels were detected in mast cells, adipocytes, placenta and esophagus. $^{42}$

Our data show that in M-CLL, COBLL1 expression is uniformly high, whereas in U-CLL patients it ranges from low to high levels. The U-CLL COBLL1-high cohort showed a strikingly worse prognosis than the COBLL1low. Shorter OS and TTST of U-CLL COBLL1-high patients remained, even after excluding patients with aberrant TP53 and the independence of COBLL1 as a prognostic factor in U-CLL for OS was proven by multivariate analysis. IGHV mutational status and COBLL1 expression thus represents a novel marker combination, which efficiently identifies patients with short OS and TTST.

We showed bimodal COBLL1 expression distribution in three independent cohorts. The U-CLL patients can be categorized according to a cut off close to a local distribution minimum which facilitates access to our marker combination by other laboratories if desirable. In addition to qRT-PCR-based assessment of COBLL1 expression, COBLL1 protein levels can in principle, be analyzed using flow cytometry. However, this would require fixation (COBLL1 is a cytoplasmic protein) and staining with a primary and secondary antibody, since there are currently no well-validated fluorescently-conjugated monoclonal antibodies.

Interestingly, functional analysis of U-CLL COBLL1-high CLL cells showed a higher response to BCR stimulation and deregulated chemotaxis in this patient cohort. This is in line with a large body of evidence showing that increased in vitro response to BCR stimuli associ- 
ates with aggressive CLL. ${ }^{43,44}$ COBLL1-high CLL cells preferentially responded by activation of BTK, SYK and ERK1/2 whereas COBLL1-low U-CLL cells induced only ERK1/2. It has been shown previously that, in healthy B cells, ERK1/2 can be efficiently phosphorylated by Iginduced BCR crosslinking even in cases when no detectable phosphorylation of BTK or SYK is seen. ${ }^{45}$ There is also some evidence that these different modes of BCR activation depend on the stimulus ${ }^{45}$ and also differ between healthy and malignant cells. ${ }^{46}$ This suggests that COBLL1 can regulate this balance and promote the BCR activation mode that involves the upstream BTK/SYK kinases.

In addition, U-CLL COBLL1-high cells exhibit impaired migration towards chemokines CCL19 and CXCL12, a phenotype very similar to aggressive CLL cells expressing ROR1 ligand Wnt-5a. ${ }^{11}$ Although we were not able to correlate WNT5A and COBLL1 expression, both studies indicate lower chemotaxis in patients with aggressive CLL and a deregulated Wnt/PCP signaling pathway. Both observations support the generally accepted view that patients with inferior prognosis often exhibit deregulated interaction with the microenvironment and with other cell types. The clear difference in TTST in U-CLL COBLL1-high patients suggests that standard therapeutic schemes do indeed have limited efficiency in this cohort. Due to their unmutated IGHV (U-CLL patients have been described as more perceptive to ibrutinib than M-CLL ${ }^{47}$ ) and high BCR responsiveness, COBLL1 can thus help to identify patients that will benefit more from the new BCR inhibitor-based therapies.

The role of COBLL1 in CLL pathogenesis and in B-cell development remains unclear. One striking observation is apparently the difference in importance of high COBLL1 in M-CLL and U-CLL. We were able to confirm previously reported uniformly high COBLL1 levels in M-CLL cells. ${ }^{48,49}$ Interestingly, M-CLL is generally more indolent than UCLL, where high COBLL1 rather counterintuitively defines patients with an inferior prognosis. Upregulation of COBLL1 in centroblasts and centrocytes compared to naive and memory cells indicates that COBLL1 is switched on during B-cell maturation in the germinal center. Together with lower IGHV germline identity in U-CLL COBLL1-high patients (compared to
U-CLL COBLL1-low patients), it suggests that upregulation of COBLL1 expression may be linked to the process of IGHV mutation. This view is also supported by the gene profiling of monoclonal B-lymphocytosis cells $(\mathrm{MBL})$ with mutated and unmutated IGHV ${ }^{50}$ where COBLL1 expression followed a similar pattern to CLL. ${ }^{48,49}$ This suggests that deregulating COBLL1 expression likely occurs prior to overt CLL, or, alternatively, points to a different origin of a U-CLL subset from a rare B-cell subset with low COBLL1 expression. This assumption is also supported by the observation that the levels of COBLL1 in U-CLL COBLL1-low samples are lower than any of the healthy Bcell populations analyzed in this study.

In summary, we identified COBLL1 as a component of the ROR1 receptor system in CLL cells. COBLL1 expression combined with IGHV hypermutation status correlates with CLL prognosis, and identifies the U-CLL COBLL1-high patients as those having an adverse disease course. U-CLL COBLL1-high cells show an increased response to $\mathrm{BCR}$ stimulation and attenuated chemotaxis, which suggests a mutual interplay between Wnt/PCP and BCR pathways in the regulation of CLL response to microenvironmental stimuli.

\section{Acknowledgments}

We wish to thank Christian Arquint and Erich A. Nigg for providing pcDNA3.1-FLAG vector, Peter Konik (University of South Bohemia, České Budějovice) for assisting with the mass spectrometry, Jana Kotašková (University Hospital Brno) for processing survival and treatment data, and Matthew Smith for language correction.

\section{Funding}

Supported by projects of the Czech Science Foundation (1716680S, 17-09525S), Ministry of Health, Czech Republic (1529793A and FNBr 65269705), by Masaryk University (MUNI/A/1106/2016, MUNI/A/0988/2016) and by projects of MEYS CR n. CEITEC 2020 (LQ1601). Also supported by the Czech Leukemia Study Group for Life (CELL). AM was financed by the programme SoMoPro, which is jointly supported by the European Union as part of the $7^{\text {th }}$ Framework Programme (FP/2007-2013, Grant Agreement n. 229603) and the South Moravian Region. VB is supported by Neuron - Fund for Support of Science.

\section{References}

1. Klein U, Tu Y, Stolovitzky GA, et al. Gene expression profiling of $\mathrm{B}$ cell chronic lymphocytic leukemia reveals a homogeneous phenotype related to memory B cells. J Exp Med. 2001;194(11):1625-1638.

2. Rosenwald A, Alizadeh AA, Widhopf G, et al. Relation of gene expression phenotype to immunoglobulin mutation genotype in $\mathrm{B}$ cell chronic lymphocytic leukemia. J Exp Med. 2001;194(11):1639-1647.

3. Masiakowski P, Carroll RD. A novel family of cell surface receptors with tyrosine kinase-like domain. J Biol Chem. 1992; 267(36):26181-26190.

4. Fukuda T, Chen L, Endo T, et al. Antisera induced by infusions of autologous AdCD154-leukemia B cells identify ROR1 as an oncofetal antigen and receptor for Wnt5a. Proc Natl Acad Sci USA. 2008; 105(8):3047-3052.
5. Baskar S, Kwong KY, Hofer T, et al. Unique cell surface expression of receptor tyrosine kinase ROR1 in human B-cell chronic lymphocytic leukemia. Clin Cancer Res. 2008; 14(2):396-404.

6. Kotašková J, Pavlová Š, Greif I, et al. ROR1based immunomagnetic protocol allows efficient separation of CLL and healthy B cells. Br J Haematol. 2016;175(2):339-342.

7. Daneshmanesh A, Hojjat-Farsangi M, Khan A, et al. Monoclonal antibodies against ROR1 induce apoptosis of chronic lymphocytic leukemia (CLL) cells. Leukemia. 2012; 26(6):1348-1355

8. Berger C, Sommermeyer D, Hudecek M, et al. Safety of targeting ROR1 in primates with chimeric antigen receptor-modified $\mathrm{T}$ cells. Cancer Immunol Res. 2015;3(2):206216.

9. Hudecek M, Schmitt TM, Baskar S, et al. The B-cell tumor associated antigen ROR1 can be targeted with $T$ cells modified to express a ROR1-specific chimeric antigen receptor. Blood. 2010;116(22):4532-4541.

10. Kaucká M, Krejčí P, Plevová K, et al. Posttranslational modifications regulate signalling by Ror1. Acta Physiol. 2011; 203(3):351-362

11. Janovska P, Poppova L, Plevova K, et al. Autocrine signaling by Wnt-5a deregulates chemotaxis of leukemic cells and predicts clinical outcome in chronic lymphocytic leukemia. Clin Cancer Res. 2016; 22(2):459. 469.

12. Khan AS, Hojjat-Farsangi $M$ Daneshmanesh AH, et al. Dishevelled proteins are significantly upregulated in chronic lymphocytic leukaemia. Tumor Biol. 2016; z37(9):11947-11957.

13. Kaucká M, Plevová K, Pavlová Š, et al. The planar cell polarity pathway drives pathogenesis of chronic lymphocytic leukemia by the regulation of B-lymphocyte migration. Cancer Res. 2013;73(5):1491-1501. 
14. Yu J, Chen L, Cui B, et al. Wnt5a induces ROR1/ROR2 heterooligomerization to enhance leukemia chemotaxis and proliferation. J Clin Invest. 2016;126(2):585-598.

15. Plevova K, Francova HS, Burckova K, et al. Multiple productive immunoglobulin heavy chain gene rearrangements in chronic lymphocytic leukemia are mostly derived from independent clones. Haematologica. 2014:99(2):329-338.

16. Baliakas P, Hadzidimitriou A, Sutton LA, et al. Clinical effect of stereotyped B-cell receptor immunoglobulins in chronic lymphocytic leukaemia: A retrospective multicentre study. Lancet Haematol. 2014; 1(2):e74-e84.

17. Baliakas P, Hadzidimitriou A, Sutton L, et al. Recurrent mutations refine prognosis in chronic lymphocytic leukemia. Leukemia. 2015;29(2):329-336.

18. Baliakas P, Iskas M, Gardiner A, et al. Chromosomal translocations and karyotype complexity in chronic lymphocytic leukemia: A systematic reappraisal of classic cytogenetic data. Am J Hematol. 2014; 89(3):249-255.

19. Malcikova J, Smardova J, Rocnova L, et al. Monoallelic and biallelic inactivation of TP53 gene in chronic lymphocytic leukemia: Selection, impact on survival, and response to DNA damage. Blood. 2009; 114(26):5307-5314

20. Pospisilova S, Gonzalez D, Malcikova J, et al. ERIC recommendations on TP53 mutation analysis in chronic lymphocytic leukemia. Leukemia. 2012;26(7):1458-1461.

21. Cajanek L, Ganii RS, Henriques-Oliveira C, et al. Tiam1 regulates the Wnt/Dvl/Rac1 signaling pathway and the differentiation of midbrain dopaminergic neurons. Mol Cell Biol. 2013;33(1):59-70

22. de Groot REA, Ganji RS, Bernatik O, et al. Huwe1-mediated ubiquitylation of dishevelled defines a negative feedback loop in the Wnt signaling pathway. Sci Signal. 2014;7(317):ra26.

23. Arquint C, Sonnen KF, Stierhof Y-D, Nigg EA. Cell-cycle-regulated expression of STIL controls centriole number in human cells. J Cell Sci. 2012;125(Pt 5):1342-1352.

24. Cervenka I, Valnohova J, Bernatik $O$, et al. Dishevelled is a NEK2 kinase substrate controlling dynamics of centrosomal linker proteins. Proc Natl Acad Sci USA. 2016; 113(33):9304-9309.

25. Bryja V, Schulte G, Arenas E. Wnt-3a utilizes a novel low dose and rapid pathway that does not require casein kinase 1-mediated phosphorylation of Dvl to activate $\beta$-catenin. Cell Signal. 2007;19(3):610-616.

26. Palomba ML, Piersanti K, Ziegler CGK, et al. Multidimensional single-cell analysis of BCR signaling reveals proximal activation defect as a hallmark of chronic lymphocytic leukemia B cells. PLoS One. 2014; 9(1):e79987.

27. $\mathrm{R}$ Development Core Team R. R: A Language and Environment for Statistical Computing. R Foundation for Statistical Computing; 2011;p409.(R Foundation for Statistical Computing; vol.1). Available from: http://www.r-project.org.

28. Zhao S, Guo Y, Shyr Y. KEGGprofile: An annotation and visualization package for multi-types and multi-groups expression data in KEGG pathway. R package version 1.14.0.

29. Krzywinski MI, Schein JE, Birol I, et al. Circos: An information aesthetic for comparative genomics. Genome Res. 2009; 19(9):1639-1645

30. Ramsay AJ, Martínez-Trillos A, Jares P, Rodríguez D, Kwarciak A, Quesada V. Next-generation sequencing reveals the secrets of the chronic lymphocytic leukemia genome. Clin Transl Oncol. 2013; 15(1):3-8

31. Kotaskova J, Tichy B, Trbusek M, et al. High expression of lymphocyte-activation gene 3 (LAG3) in chronic lymphocytic leukemia cells is associated with unmutated immunoglobulin variable heavy chain region (IGHV) gene and reduced treatment-free survival. J Mol Diagn. 2010;12(3):328-334.

32. Zamò A, Ott G, Katzenberger $T$, et al. Establishment of MAVER-1 cell line, a model for leukemic and aggressie mantle cell lymphoma. Haematologica. 2006; 91(1):40-47.

33. Paganoni S, Ferreira A. Neurite extension in central neurons: a novel role for the receptor tyrosine kinases Ror1 and Ror2. J Cell Sci. 2005;118(Pt 2):433-446.

34. Cui B, Ghia EM, Chen L, et al. High-level ROR1 associates with accelerated diseaseprogression in chronic lymphocytic leukemia. Blood. 2016;128(25):2931-2940.

35. Döhner H, Stilgenbauer S, Benner A, et al. Genomic Aberrations and Survival in Chronic Lymphocytic Leukemia. N Engl J Med. 2000;343(26):1910-1916.

36. Hamblin TJ, Davis ZA, Oscier DG Determination of how many immunoglobulin variable region heavy chain mutations are allowable in unmutated chronic lymphocytic leukaemia - Long-term follow up of patients with different percentages of mutations. Br J Haematol. 2008;140(3):320-323.

37. ten Hacken E, Burger JA. Microenvironment interactions and B-cell receptor signaling in Chronic Lymphocytic Leukemia: Implications for disease pathogenesis and treatment. Biochim Biophys Acta. 2016;1863(3):401-413.

38. Burger JA. Chemokines and chemokine receptors in chronic lymphocytic leukemia (CLL): From understanding the basics towards therapeutic targeting. Semin Cancer Biol. 2010:20(6):424-430.

39. Carroll EA, Gerrelli D, Gasca S, et al.
Cordon-bleu is a conserved gene involved in neural tube formation. Dev Biol. 2003, 262(1):16-31.

40. Gordon GJ, Jensen R V, Hsiao LL, et al. Using gene expression ratios to predict outcome among patients with mesothelioma. J Natl Cancer Inst. 2003;95(8):598-605.

41. Han SH, Kim SH, Kim HJ, et al. Cobll1 is linked to drug resistance and blastic transformation in chronic myeloid leukemia. Leukemia. 2017;31(7):1532-1539.

42. Benita Y, Cao Z, Giallourakis C, Li C, Gardet A, Xavier RJ. Gene enrichment profiles reveal T-cell development, differentiation, and lineage-specific transcription factors including ZBTB25 as a novel NF-AT repressor. Blood. 2010;115(26):5376-5384.

43. Morabito F, Cutrona G, Gentile M, et al Prognostic relevance of in vitro response to cell stimulation via surface $\operatorname{IgD}$ in binet stage a CLL. Br J Haematol. 2010, 149(1):160-163.

44. Lanham S, Hamblin T, Oscier D, Ibbotson R, Stevenson F, Packham G. Differential signaling via surface IgM is associated with $\mathrm{VH}$ gene mutational status and $\mathrm{CD} 38$ expression in chronic lymphocytic leukemia. Blood. 2003:101(3):1087-1093.

45. Irish JM, Czerwinski DK, Nolan GP, Levy R. Kinetics of B Cell Receptor Signaling in Human B Cell Subsets Mapped by Phosphospecific Flow Cytometry. J Immunol. 2006;177(3):1581-1589.

46. Irish JM, Czerwinski DK, Nolan GP, Levy R. Altered B-cell receptor signaling kinetics distinguish human follicular lymphoma B cells from tumor-infiltrating nonmalignant B cells. Blood. 2006;108(9):3135-3142

47. Thompson PA, O'Brien SM, Wierda WG, et al. Complex karyotype is a stronger predictor than del(17p) for an inferior outcome in relapsed or refractory chronic lymphocytic leukemia patients treated with ibrutinibbased regimens. Cancer. 2015; 121(20):3612-3621.

48. Abruzzo LV, Barron LI, Anderson K, et al. Identification and validation of biomarkers of $\operatorname{IgV}(\mathrm{H})$ mutation status in chronic lymphocytic leukemia using microfluidics quantitative real-time polymerase chain reaction technology. J Mol Diagn. 2007, 9(4):546-555

49. Plesingerova $\mathrm{H}$, Librova $\mathrm{Z}$, Plevova $\mathrm{K}$, et al. COBLL1, LPL and ZAP70 expression defines prognostic subgroups of chronic lymphocytic leukemia patients with high accuracy and correlates with IGHV mutational status. Leuk Lymphoma. 2017; 58(1):70-79

50. Morabito F, Mosca L, Cutrona G, et al Clinical monoclonal B lymphocytosis versus rai 0 chronic lymphocytic leukemia: A comparison of cellular, cytogenetic, molecular, and clinical features. Clin Cancer Res. 2013;19(21):5890-5900. 